\title{
Plasma interferon- $\gamma$-inducible protein 10 (IP-10) levels correlate with disease severity and paradoxical reactions in extrapulmonary tuberculosis
}

\author{
Isabelle Suárez ${ }^{1,2} \cdot$ Samuel Rohr $^{1} \cdot$ Melanie Stecher ${ }^{1,2} \cdot$ Clara Lehmann $^{1,2,3} \cdot$ Sandra Winter $^{1,3} \cdot$ Norma Jung $^{1,2}$. \\ Vanessa Priesner $^{1,2} \cdot$ Melanie Berger $^{5} \cdot$ Christoph Wyen $^{1,6} \cdot$ Max Augustin $^{1,3} \cdot$ Jakob J. Malin $^{1,2,3} \cdot$ Julia Fischer $^{1,2,3}$. \\ Carola Horn $^{1,3}$. Florian Neuhann ${ }^{4,9,10} \cdot$ Michael Püsken $^{8}$. Georg Plum ${ }^{7}$. Gerd Fätkenheuer ${ }^{1,2}$ • Jan Rybniker ${ }^{1,2,3}$ (D)
}

Received: 11 September 2020 / Accepted: 21 October 2020 / Published online: 2 November 2020

(c) Springer-Verlag GmbH Germany, part of Springer Nature 2020

\begin{abstract}
Background With 1.5 million deaths worldwide in 2018, tuberculosis (TB) remains a major global public health problem. While pulmonary TB (PTB) is the most common manifestation, the proportion of extrapulmonary TB (EPTB) is increasing in low-burden countries. EPTB is a heterogeneous disease entity posing diagnostic and management challenges due to the lack of reliable biomarkers. In this study, we prospectively evaluated clinical data and treatment response which were correlated with different biomarkers.

Methods The study was conducted at the University Hospital of Cologne. 20 patients with EPTB were enrolled. We analyzed plasma interferon- $\gamma$-inducible protein 10 (IP-10) levels in plasma by ELISA for up to 12 months of treatment. In addition, the QuantiFERON $^{\circledR}$-TB Gold Plus ( QFT $^{\circledR}$ Plus) test was performed during the course of treatment. Clinical data were assessed prospectively and correlated with $\mathrm{QFT}^{\circledR}$ Plus and IP-10 levels.

Results Plasma IP-10 levels were found to be significantly increased $(p<0.001)$ in patients with extensive disease compared to patients with limited disease (cervical lymph node TB) or healthy controls. In patients with clinically confirmed paradoxical reaction (PR), a further increase of IP-10 was noted. IFN- $\gamma$ measured by the QFT ${ }^{\circledR}$ Plus test did not decrease significantly during the course of treatment. Of note, in four EPTB patients (20\%) without radiographic pulmonary involvement, sputum culture was positive for Mycobacterium tuberculosis.

Conclusion Our data demonstrate that IP-10 may be a valuable biomarker for estimation of disease severity in EPTB and monitoring of the disease course in extensive forms. However, IP-10 may be less suitable for diagnosis and monitoring of EPTB patients with limited disease. The $\mathrm{QFT}^{\circledR}$ Plus test does not appear to be a suitable marker for therapy monitoring. Sputum should be examined in EPTB patients even in case of normal diagnostic imaging of the chest.
\end{abstract}

Keywords Extrapulmonary tuberculosis $\cdot$ Biomarker $\cdot$ IP-10 $\cdot$ Paradoxical reaction · QuantiFERON ${ }^{\circledR}$-TB gold plus test . EPTB

\section{Introduction}

About 10 million people contracted tuberculosis (TB) in 2018 [37]. The burden of disease varies enormously between different regions [37]. Management of TB in low-burden

Supplementary information is available for this paper at https:// doi.org/10.1007/s15010-020-01541-1.

Jan Rybniker

jan.rybniker@uk-koeln.de

Extended author information available on the last page of the article countries means increasingly focusing on extrapulmonary manifestations of the disease, since a rise in the proportions of extrapulmonary TB (EPTB) of up to $50 \%$ has been observed in some areas $[6,20]$ over the past decades. Despite this observation, EPTB remains largely neglected by public health and clinical research compared to pulmonary TB (PTB). There are only few prospective studies focusing on EPTB, although the correct diagnosis and optimal treatment of EPTB is challenging. Microbiological confirmation of suspected EPTB often requires tissue biopsies, whereas treatment monitoring is usually based on clinical findings and imaging due to the lack of suitable biomarkers $[1,9]$. 
Paradoxical reactions (PR) with enlargement of lesions despite effective treatment occur frequently, and can cause uncertainties regarding the treatment response that may eventually lead to prolonged therapy courses.

In PTB, the best studied biomarker for successful treatment is conversion to negative sputum culture [15], which is not applicable to most EPTB cases where repetitive invasive tissue sampling is not recommended. Thus, validation of alternative biomarkers for treatment response is urgently needed in EPTB to shorten treatment duration and to ensure rapid detection of treatment failure, often resulting in the development of drug resistance. Interferon (IFN)- $\gamma$ inducible protein 10 (IP-10), a pro-inflammatory chemokine secreted by a variety of immune cells such as monocytes, neutrophils, macrophages, and endothelial cells, has been proposed as surrogate for treatment response in PTB [14, 36]. IP-10 levels are reported to be higher and detected more reproducibly than IFN- $\gamma$ and therefore IP-10 is considered a candidate biomarker for control of TB. Recent data revealed a significant decline of IP-10 measured in plasma or on dry plasma spots (DPS) in successfully treated TB patients [13].

The QuantiFERON ${ }^{\circledR}$-TB Gold Plus (QFT ${ }^{\circledR}$ Plus) assay, a highly standardized assay for detection of latent TB infections (LTBI) diagnostics [24, 31, 35], detects interferon- $\gamma$ $(\mathrm{IFN}-\gamma)$ released from peripheral blood monocytes stimulated with specific $M t b$ antigens. At present, the assay is not recommended for the purpose of treatment monitoring. However, some studies indicate that the latest test generation may provide a tool for treatment monitoring in PTB. Therefore, our aim within this study was to evaluate IP-10 and the $\mathrm{QFT}^{\circledR}$ Plus assay in the course of treatment in patients with EPTB and to prospectively analyze clinical characteristics of EPTB patients.

\section{Methods}

\section{Study participants}

Since August 2018 patients with confirmed diagnosis of EPTB presenting at the University Hospital of Cologne, Germany, were enrolled in a prospective, monocentric study for EPTB (Cologne EX-TB study) after obtaining informed consent. EPTB was diagnosed based on a positive culture, positive acid-fast bacilli (AFB) microscopy or PCR for $M$. tuberculosis $(\mathrm{Mtb})$ complex, detection of extrapulmonary lesions in imaging (e.g., CT, MRI, X-rays, ultrasound) and histopathological findings. Sputum (3 days in a row) and urine samples were taken from every patient at baseline (for $M t b$ culture, microscopy and PCR). Blood samples were collected in all patients at baseline, after 1, 3, 6 months, and in cases with prolonged treatment duration also at 9 and 12 months of treatment. The study was approved by the Ethics Committee of the University of Cologne (18-079).

\section{Paradoxical reaction}

Paradoxical reaction (PR) was defined as clinical worsening (e.g., fever, pain) or a radiographic increase in the size of previously identified lesions or the appearance of new lesions despite microbiologically appropriate TB therapy in the absence of disease relapse or the presence of another diagnosis $[4,10]$.

\section{Imaging}

Imaging was performed to assess general organ involvement at baseline and treatment response, according to the involved organ. Thus, in seven patients with cervical lymph node TB, sonography of all lymph nodes was performed every 3 months. Furthermore, sonography was performed in two cases with abdominal TB every 3 months for follow-up. In three patients with bone affection, magnetic resonance imaging (MRI) was carried out every 3-6 months, and in the presence of other manifestations (e.g., mediastinal lymph nodes, pleural involvement etc.), CT scans were performed every 3-6 months. To determine the extent of the disease, a positron emission tomography (PET)-CT was performed in two cases. We distinguished between cases with only localized organ involvement, consisting of 1-2 cervical lymph nodes (limited disease), and cases with a more disseminated form of TB (extensive disease).

\section{Statistical methods}

Data processing and statistical analysis were performed using the software GraphPad Prism Version 8.0. Patient characteristics were reported as absolute numbers with percentage and median with interquartile range (IQR) as appropriate. Non-parametrical and parametrical statistical methods were used. Friedman test or one-way ANOVA was applied for measuring differences during treatment. For groupwise comparison two-way ANOVA or Mann-Whitney $U$ test was applied and for dependent variables two-tailed Wilcoxon matched pair test. $p=0.05$ was considered statistically significant.

\section{Plasma IP-10 enzyme-linked immunosorbent assay (ELISA)}

Plasma IP-10 was determined in duplicate by using a commercially available ELISA kit (Sigma Aldrich, Human IP-10/CXCL10 ELISA Kit, for serum, plasma, and cell culture supernatants, Product Number RAB0119) according to the manufacturer's instructions. The IP-10 levels were 
measured at baseline ( \pm 7 days of treatment initiation) and after 3 and 6 months (where available 9 and 12 months after treatment initiation). The minimum detectable level for IP-10 was 8 pg/ml.

\section{QuantiFERON ${ }^{\circledast}-$ TB Gold Plus assay}

The $\mathrm{QFT}^{\circledR}$ Plus assay was performed at baseline and after 1,3 and 6 months of treatment. The QFT $^{\circledR}$ Plus assay was performed as per the manufacturer's instructions with an incubation time between 16 and $24 \mathrm{~h}$ at $37{ }^{\circ} \mathrm{C}$. Tubes were centrifuged, the plasma removed and the amount of IFN-y (ILI/ml) measured by ELISA. The antigens used in the QFT $^{\circledR}$ Plus assay are a peptide cocktail simulating the proteins ESAT-6, CFP-10 and TB7.7(p4) (contained by both tubes Antigen 1 and 2) [26]. A recombinant human IFN-y standard, which had been assayed against a reference IFN-y preparation (NIH Ref: GxgO 1-902-535), was used to calibrate a four-point standard curve and interpret sample optical density into estimated IFN-y (IU/ml). A test was considered positive for an IFN-y response if the negative control tube (NIL) value was lower than $8.0 \mathrm{IU} / \mathrm{ml}$ plus the IFN-y IU/ml value of the TB antigen minus the NIL value was greater or equal to $25 \%$ of the NIL value plus greater or equal to $0.35 \mathrm{IU} / \mathrm{ml}$.

\section{Results}

\section{Study subjects}

Since August 2018, a total of 20 patients were included in this study. Seven patients (35\%) were diagnosed with isolated cervical lymph node TB. 14 patients (64\%) suffered from a more severe form of TB with, in some cases, dissemination of the disease to different organ sites (suppl. Table 1). Culture was positive for Mycobacterium tuberculosis (17) or Mycobacterium bovis (2) in all cases except for one. In this patient, diagnosis was based on clinical suspicion, histology findings and positive $\mathrm{QFT}^{\circledR}$ Plus test. In 13 patients (65\%), the diagnosis was obtained by lymph node biopsy, three by bone biopsy, two by pleura puncture, one by peritoneal biopsy and one by liver biopsy. All patients were examined for $M t b$ sputum positivity by microscopy, PCR and culture. In five (25\%) patients, $M t b$ was detected in a sputum sample by PCR and culture despite negative imaging results for pulmonary involvement (3 X-rays and $2 \mathrm{CT}$ scans). Sputum smear was microscopically AFB negative in all of our patients. 16 patients received a standard four-drug TB regimen (INH, RMP, PZA, EMB), whereas two patients received a three-drug regimen due to intrinsic PZA resistance in M.bovis species. Treatment had to be modified due to isoniazid resistance in two patients (RMP, PZA, EMB, MXN).

\section{Imaging}

In two cases, PET-CT was performed at baseline demonstrating FDG-avid lesions involving mainly lymph nodes. In patients with limited disease, a reduction in lymph node size was observed in all patients within 6-9 months of TB therapy. PR occurred in four patients with extensive disease and was suspected in one patient with limited disease due to repeated fistulas. Corticosteroids were administered in two patients with suspected PR which led to decrease of lesion size. The remaining patients were clinically monitored closely and the PR appeared to be self-limiting. Figure 1 exemplarily shows two patients with suspected PR due to increasing lesion size on follow-up imaging after an excellent initial clinical response on adequate TB therapy. None of these patients with PR were sputum culture positive.

\section{Plasma IP-10 levels}

The median IP-10 plasma level was $405 \mathrm{pg} / \mathrm{ml}$ (197.80-1547.00) in extensive TB vs. $68 \mathrm{pg} / \mathrm{ml}$ (23.42-125.30) in limited disease at baseline, therefore IP-10 levels were found to be significantly higher $(p<0.001)$ in patients with extensive disease (Fig. 2). Baseline plasma IP-10 levels in patients with limited disease did not differ from levels found in plasma from healthy controls despite using a highly sensitive ELISA test system. Sputum culture positivity was not associated with elevated IP-10 levels $(p=0.8854)$. Of note, in two patients with limited disease sputum culture came back positive, but baseline IP-10 levels were low. In eight patients with extensive disease, IP-10 levels were longitudinally observed during the course of treatment. Four patients showed a significant decline of IP-10 levels corresponding to clinical and radiographic improvement during 6 months of treatment $(p<0.001)$. In the other four patients, IP-10 levels either remained high or increased further (Fig. 3). These were precisely the patients with extensive TB that were clinically and by imaging diagnosed with a PR (Fig. 3). Three of these patients, who experienced a PR, did not have any known concomitant disease. One patient was HIV positive.

\section{IP-10 levels in correlation to other inflammatory markers}

CRP was referred to as not elevated when the values were below $5 \mathrm{mg} / \mathrm{l}$. 80\% (16) of our patients had elevated CRP at baseline. The median baseline CRP was $23.5 \mathrm{mg} / \mathrm{l}$ (IQR 6.9-77.4; $N=20$ ), after 1 month of treatment median CRP was $7.7 \mathrm{mg} / \mathrm{l}$ (IQR $3.4-66.1 ; N=20$ ), after 3 months of 

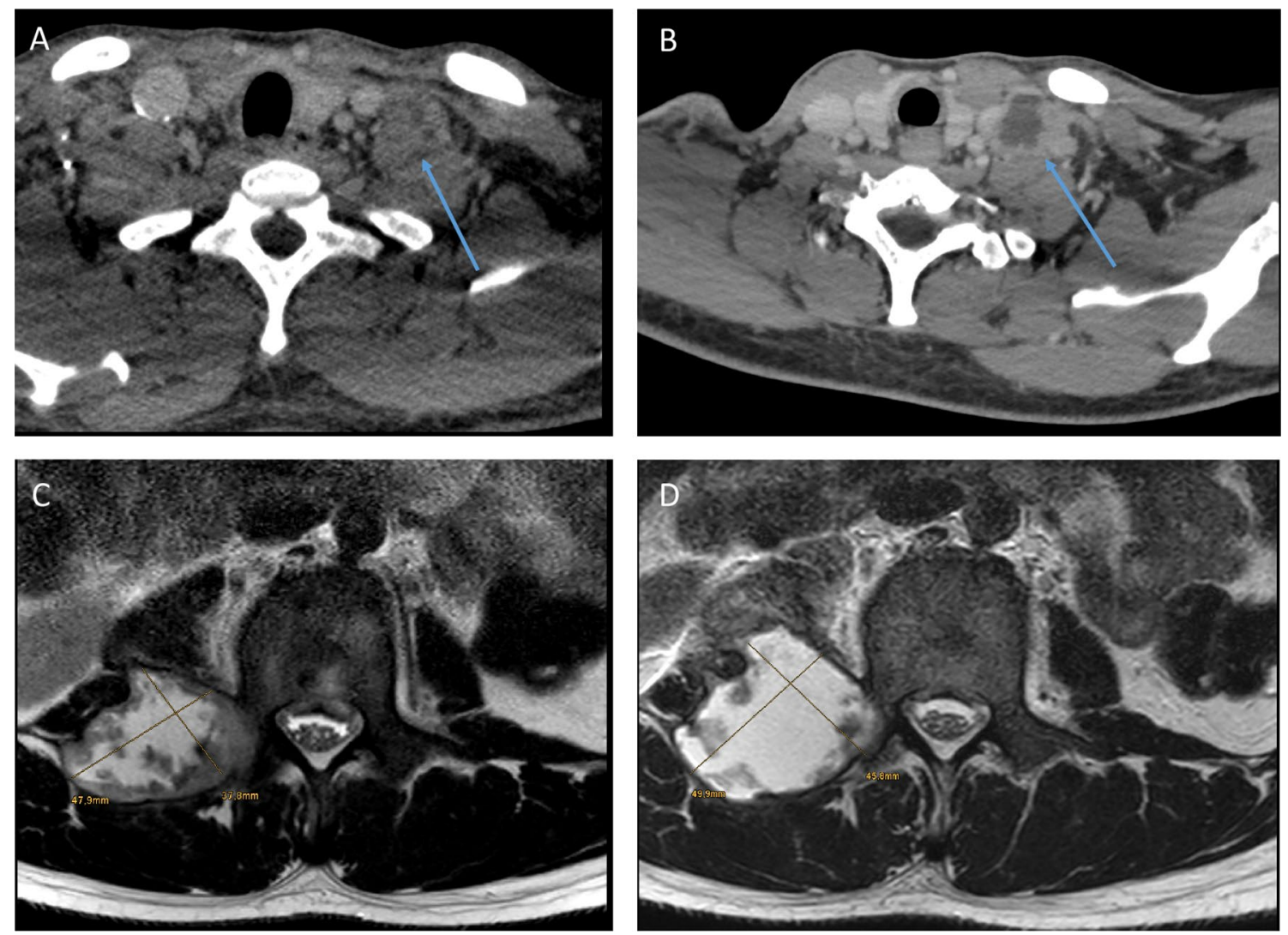

Fig. 1 Paradoxical reaction-CT scan with contrast medium: a cervical lymph node at baseline and $\mathbf{b}$ after 4 months of treatment showing increasing cervical lymph nodes in a HIV-positive patient with

disseminated TB. MRI of the spine (T2 TSE axial): c paravertebral abscess at baseline and $\mathbf{d}$ after 2 months of treatment showing an increase of lesion size

In patients with extensive disease, median CRP was significantly higher than in limited disease $(p=0.0059)$ at baseline [43.90 mg/l (IQR 20.1-89.7; $N=13$ ) vs. $6.6 \mathrm{mg} / \mathrm{l}$ (IQR 1.6-9.2; $N=7$ )]. There was a significant correlation between CRP and IP-10 levels ( $p<0.001 ; p=0.69)$ at baseline, but a correlation was not seen any more after 3 and 6 months of treatment $(p=0.58 ; p=0.75)$. Of note, three out of four patients showed a rapid decline and normalization of CRP despite clinical and radiographic signs of PR, which stands in contrast to plasma IP-10 levels (Fig. 3). The median baseline erythrocyte sedimentation rate (ESR) was $52 \mathrm{~mm} / \mathrm{h}$ (IQR 15.2-78.0). There was no significant difference between limited and extensive disease $(p=0.14)$ concerning ESR at baseline. Furthermore, no significant correlation could be shown between ESR and IP-10 levels $(p=0.14)$ at baseline.

\section{QuantiFERON-TB Gold ${ }^{\circledR}$ assay}

treatment median CRP was $6.00 \mathrm{mg} / \mathrm{l}$ (IQR 1.7-12.6; $N=17)$ and after 6 months of treatment median CRP was $3.5 \mathrm{mg} / \mathrm{l}$ (IQR 0.8-13.9; $N=15$ ) in our cohort (Suppl. Fig. 1). After 1 month of treatment, $11(55 \%)$ patients exhibited normalized CRP values $(p=0.0103)$.

In 15 patients, $\mathrm{QFT}^{\circledR}$ Plus assay was analyzed in the course of treatment. No significant decline of Antigen tube 1 $(p=0.9713)$ or $2(p=0.4698)$ in IFN- $\gamma$ level (Fig. 4$)$ was detectable. Baseline values and values gathered during the 


\section{Extensive disease without paradoxical reactions}

Patient A

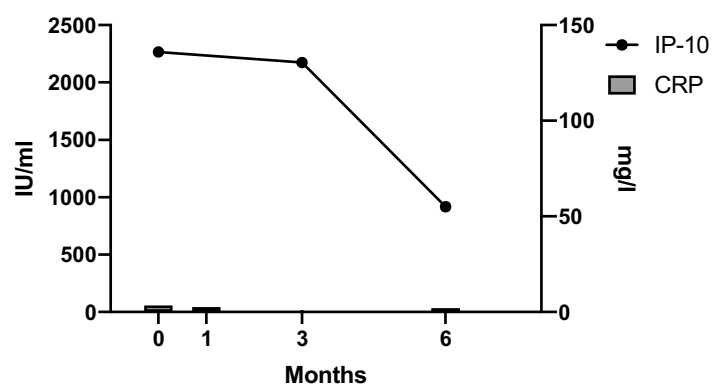

Patient C

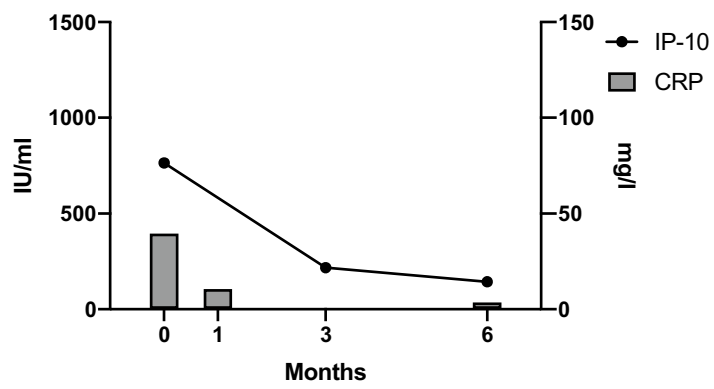

Patient B

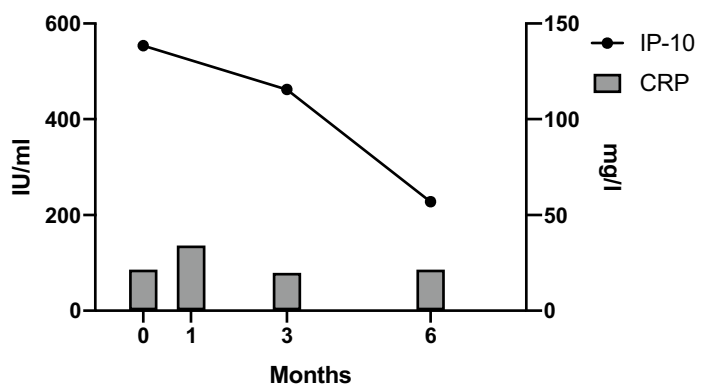

Patient D

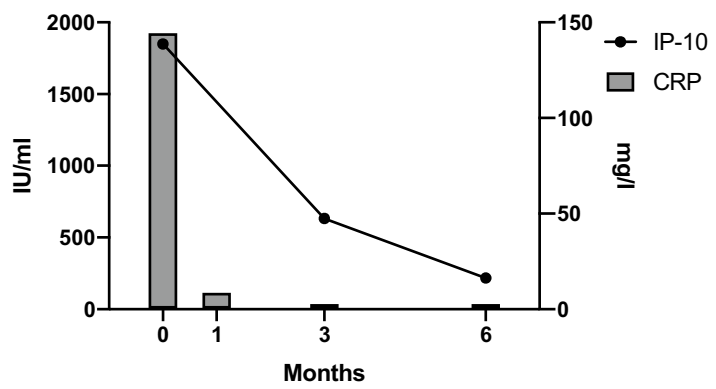

\section{Extensive disease with paradoxical reactions}
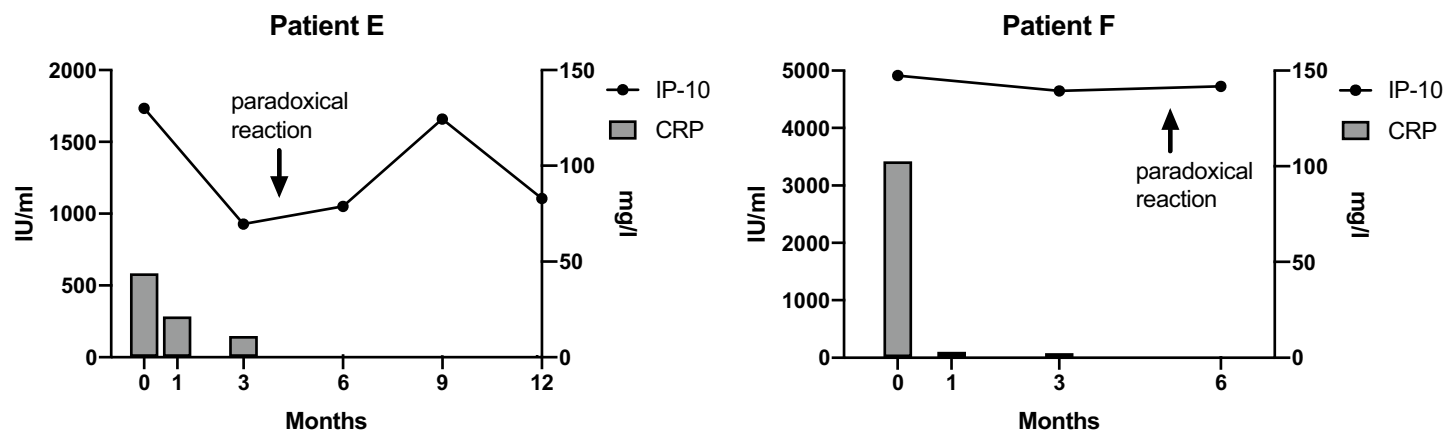

Patient G
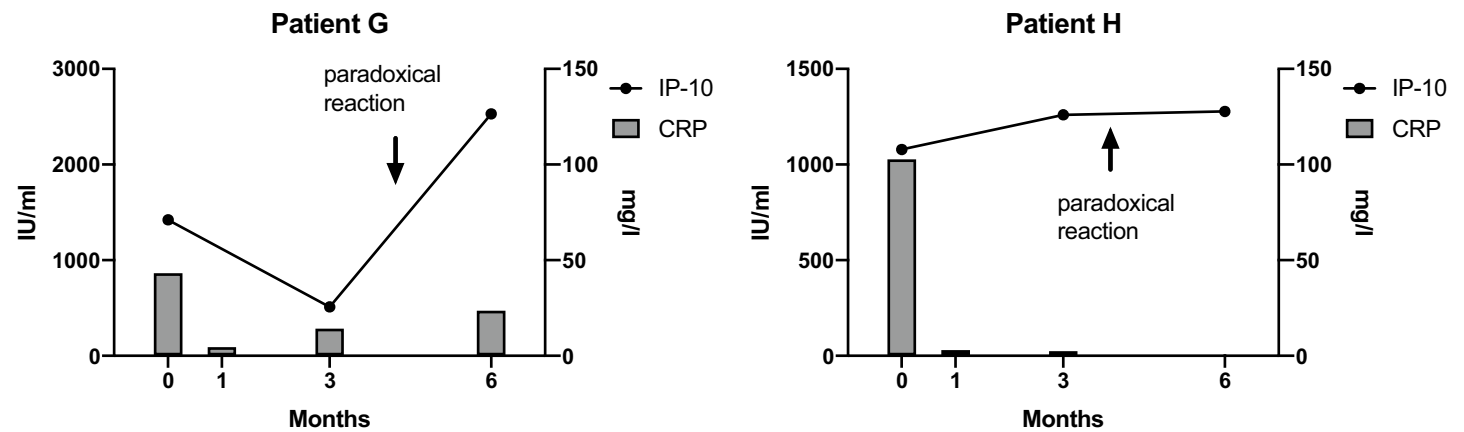

Fig. 3 IP-10 levels and CRP at different time points in the course of treatment in four patients without and four patients with paradoxical reaction 


\section{Antigen Tube 1}

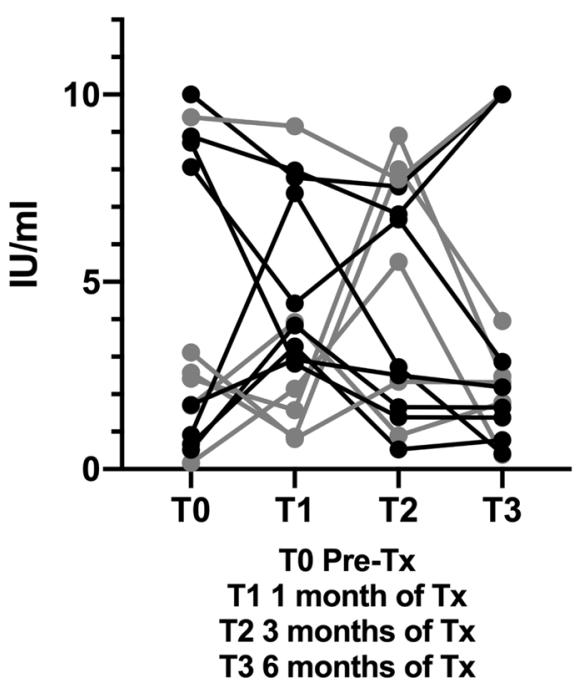

Antigen Tube 2

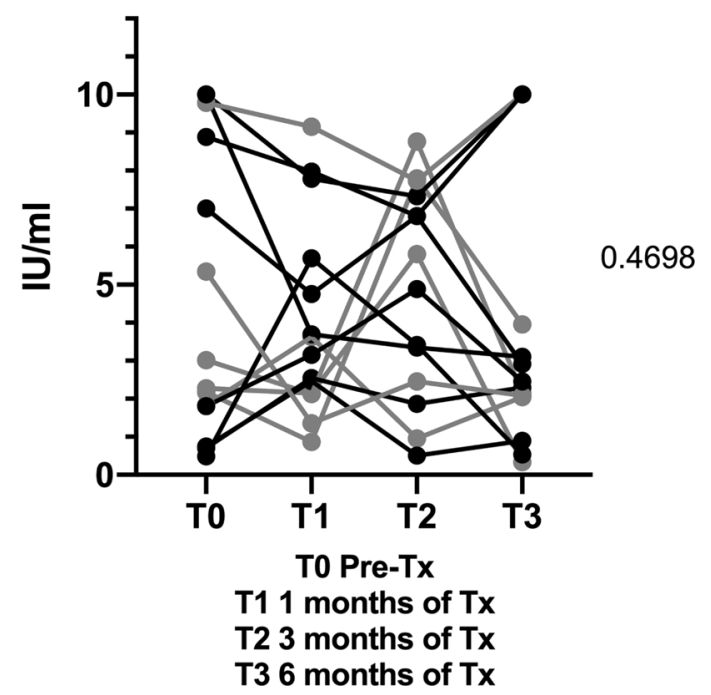

Fig. 4 QuantiFERON-TB Gold Plus ${ }^{\circledR}$ IFN- $\gamma$ levels pre-treatment and after 4 weeks, 3 months and 6 months of treatment. Limited disease (gray) and extensive disease (black)

treatment course did not correlate with the extent of disease or presence of PR (Fig. 4).

\section{Discussion}

We conducted a prospective observational study to describe the clinical course of EPTB along with biosampling, which allowed for longitudinal evaluation of different biomarkers including IP-10 and the most recent version of the QFT ${ }^{\circledR}$ Plus assay. All patients were examined for $M t b$ sputum positivity even though imaging (Chest X-ray or CT) did not show pulmonary involvement. In five (25\%) patients with normal chest radiography (3 X-ray and 2 CT scan), $M t b$ could be detected in a sputum sample by PCR and culture. This important observation indicates that even in the case of solely extrapulmonary involvement determined by radiography, sputum specimen should be examined for $M t b$ in suspected EPTB. This may facilitate and accelerate the diagnosis and may exclude any possible risk of infectivity. Subsequently, sputum culture conversion could be used for monitoring of treatment response in these patients. In a retrospective study involving 248 EPTB patients, ElHasmi et al. could show similar findings, where $23 \%$ of their patients with EPTB and normal chest radiographs revealed positive sputum culture results [7]. However, in most EPTB patients, clinical management is particularly challenging since response to therapy needs to be evaluated by imaging. Non-specific inflammatory parameters such as CRP and ESR may be elevated at the onset of the disease. In our cohort, median CRP was significantly higher in patients with extensive forms of EPTB than in limited disease at baseline $(p=0.0059)$. After 1 month of therapy, the CRP was normalized in about half of the patients (55\%). This shows that CRP can be used to evaluate initial response to therapy (Figs. 3 and Suppl. Fig. 1). Due to the rapid decline in the course of treatment, it is not a useful marker for determining the treatment duration in TB in general.

IP-10 has been widely studied as a biomarker for TB. Several studies considered IP-10 to be valuable in the diagnosis of TB or the differentiation between LTBI and active TB (ATB) when determined in the cell supernatant after stimulation with Mtb-specific antigens ( $\mathrm{Ag}$ ) [23, 27, 28, 36]. However, so far, no consistent cutoff values have been defined. In a meta-analysis by Qiu et al., studies performed with plasma IP-10 or IP-10 detected in the supernatant of Ag-stimulated whole blood were included. An overall poor diagnostic value with sensitivity of $72 \%$ could be shown for the discrimination between LTBI and ATB for stimulated and unstimulated IP-10 measurement [27, 28]. Our data indicate that, despite using a highly sensitive ELISA test system, patients with proven ATB but limited disease show very low plasma IP-ten levels below $100 \mathrm{pg} / \mathrm{ml}$ and values do not differ from those found in the healthy control (Fig. 2). These patients would not have been diagnosed with ATB by plasma IP-10 measurement alone.

Plasma IP-10 has also been recognized as a biomarker for therapy monitoring of PTB and EPTB [14, 23, 33]. For PTB, it could be shown that the initial IP-10 levels correlate with the sputum bacterial load evaluated by microscopy [14]. Significant changes in IP-10 levels between the time of diagnosis and treatment completion were observed. Patients 
with relapsing disease showed an increase of IP-10 [13, 14, 33]. Hoel et al. investigated whether IP-10 quantified in plasma and in dried plasma or blood spots could be used to monitor treatment efficacy of EPTB patients. Elevated levels were observed in the majority of patients, and a decline was seen after 2 months and from baseline to the end of treatment. However, in this study, the extent of the disease was not evaluated and no comparison was made between different grades of dissemination [13]. In our study, plasma IP-10 does not seem to be useful for treatment monitoring in EPTB patients with limited disease such as isolated cervical lymph node TB, since IP-10 levels were too low at baseline. However, in patients with extensive disease, our data suggest that IP-10 is elevated at baseline and decreases over the full course of treatment (Fig. 3), whereas CRP values rapidly decline during the first weeks of treatment. Thus, IP-10 may be of use in determining the treatment duration in EPTB. This hypothesis should nevertheless be considered with caution, since we only observed four patients over a period of 6 months.

In four (20\%) patients with extensive disease and radiologically confirmed PR, there was no decline of plasma IP-10 (Fig. 3). PR or TB immune reconstitution inflammatory syndrome (TB-IRIS), which is often used synonymously [21], is frequently observed in EPTB [10] occurring in up to $25 \%$ of HIV-negative patients. In HIV-positive patients, the rates are even higher [2]. It is most frequently observed in HIV/TB coinfected patients with low CD4 cell count after initiation of antiretroviral therapy [3]. In contrast, an 'unmasking IRIS' or HIV-IRIS is an immune response against a pathogen that has not caused any obvious clinical disease before the initiation of ART [29]. IP-10 plays an important role in HIV infection and is associated with disease progression [22]. In PR or TB-IRIS, a cytokine release syndrome is suspected to be the underlying mechanism [32]. Interestingly, CRP did not increase when PR was suspected in our cohort (Fig. 3). Plasma IP-10 is increased in numerous infectious diseases, such as hepatitis C [12], bacteremia [8] and in other inflammatory conditions [19]. In patients with rheumatoid arthritis, IP-10 was suggested as a useful tool to determine disease activity and disease progression which was superior to common inflammatory markers such as CRP [34] due to a better correlation with clinical disease activity scores [34]. Yang et al. analyzed in detail the differences in cytokine expression profiles in critically ill, severe and moderate COVID-19 cases and observed that, among other cytokines, IP-10 is highly associated with disease severity and can be used as an excellent predictor for disease progression [38]. Therefore, the determination of IP-10 levels in patients with COVID-19 in early clinical stages may provide useful information for treatment strategies. Similar findings were described for Ebola virus disease [17]. IP-10 could also be useful in other chronic progressive infections with inflammatory exacerbations where treatment responses may be difficult to monitor. One example is leprosy, an infectious disease caused by $M$. leprae where assessment of acute inflammatory reactions may be difficult only by clinical evaluation as it is the case in TB with assumed PR [18]. IP-10 values that remain steady or increase during the treatment course may predict the occurrence of PR. Thus, IP-10 could be useful in personalized treatment approaches with immunomodulatory drugs such as corticosteroids or other host-directed therapies [11, 30]. Persistently high IP-10 levels could also indicate a lack of treatment response, possibly due to drug resistance. It seems not possible to differentiate between PR and inadequate treatment using IP-10 alone and additional information on treatment adherence and drug resistance of the respective strain is required for interpretation. In addition to IP-10, we also investigated the QFT $^{\circledR}$ Plus assay in the course of treatment. Recent publications reported that the $\mathrm{QFT}^{\circledR}$ Plus assay may be useful for treatment monitoring in PTB [16] [25]. This interferon- $\gamma$ release assay (IGRA) detects IFN- $\gamma$ secreted by CD8- and CD4positive T cells [16]. Kamada et al. described a statistically significant decrease of IFN- $\gamma$ in PTB within the course of treatment, which is different from studies using the previous version of the QuantiFERON-TB Gold assay [5]. We could not reproduce these findings for EPTB in our study (Fig. 3); there was no significant decrease of the measured IFN- $\gamma$ levels (Fig. 4$)$ in Antigen tube $1(p=0.97)$ or $2(p=0.46)$ over the treatment course of 6 months.

In conclusion, our data show that IP-10 levels are very low in limited EPTB and thus do not seem to be suitable for diagnostics or to monitor the course of the disease in these patients. In more extensive forms of EPTB, IP-10 may be of value for treatment monitoring and for identification of PR where the QuantiFERON-TB Gold assay is of limited use. IP-10-guided host-directed therapy with corticosteroids could be an option for improved management of PR in culture-confirmed EPTB cases which requires further evaluation in prospective studies with larger patient numbers. Complete sputum diagnostics should be performed in every patient with suspected or otherwise confirmed EPTB.

Acknowledgements We thank Ute Sandaradura, Jason Chhen and Eleonore Thomas for support in carrying out the study. We thank Tanja Hoffmann for reporting microbiologically confirmed TB cases. We thank Jörg Janne Vehreschild and Stephanie Heinen for the collaboration and support in using the Laboratory Information and Management System HEnRY (HIV Engaged Research Technology).

Author contributions JR and IS initiated the idea and developed the first draft outline. JR and IS wrote the manuscript. SR, IS and MS collected data and analyzed data. Subsequent drafts of the manuscript were developed by CL, SW, NJ, VP, MB, CW, MA, JJM, MP, GP and GF. All authors were involved in patient care. All authors contributed to all sections relevant to their experience and helped finalize the text and content. 
Funding JR received funding from the Thematic Translational Unit Tuberculosis (TTU TB, Grant numbers TTU 02.806 and 02.905) of the German Center of Infection Research (DZIF). Financial support was also received from the German Research Foundation (DFG RY159) and the Center for Molecular Medicine Cologne (ZMMK-CAP8). IS received funding by the German Center for Infection Research (DZIF) (Grant number TI 07.001_SUAREZ_00).

\section{Compliance with ethical standards}

Conflicts of interest All authors declare no conflict of interest.

\section{References}

1. Al-Tawfiq JA, Al-Khatti A. Successful treatment of extra-pulmonary tuberculosis presenting concomitantly with acute myeloid leukemia. Infection. 2019;47:869-74.

2. Breen RA, Smith CJ, Bettinson H, Dart S, Bannister B, Johnson MA, Lipman MC. Paradoxical reactions during tuberculosis treatment in patients with and without HIV co-infection. Thorax. 2004;59:704-7.

3. Breton G, Duval X, Estellat C, Poaletti X, Bonnet D, Mvondo Mvondo D, Longuet P, Leport C, Vilde JL. Determinants of immune reconstitution inflammatory syndrome in HIV type 1-infected patients with tuberculosis after initiation of antiretroviral therapy. Clin Infect Dis. 2004;39:1709-12.

4. Brown CS, Smith CJ, Breen RA, Ormerod LP, Mittal R, Fisk M, Milburn HJ, Price NM, Bothamley GH, Lipman MC. Determinants of treatment-related paradoxical reactions during antituberculosis therapy: a case control study. BMC Infect Dis. 2016;16:479.

5. Denkinger CM, Pai M, Patel M, Menzies D. Gamma interferon release assay for monitoring of treatment response for active tuberculosis: an explosion in the spaghetti factory. J Clin Microbiol. 2013;51:607-10.

6. ECDC. 2020. 'Tuberculosis surveillance and monitoring in Europe 2020-2018 data'.

7. El-Hazmi MM, Al-Otaibi FE. Predictors of pulmonary involvement in patients with extra-pulmonary tuberculosis. J Family Community Med. 2012;19:88-92.

8. Fuchs A, Gotta V, Decker ML, Szinnai G, Baumann P, Bonhoeffer J, Ritz N, Paed study group Pro. Cytokine kinetic profiles in children with acute lower respiratory tract infection: a post hoc descriptive analysis from a randomized control trial. Clin Microbiol Infect. 2018;24:1341.e1-41.e7.

9. Gambhir S, Ravina M, Rangan K, Dixit M, Barai S, Bomanji J, T. B. Consortium International Atomic Energy Agency Extrapulmonary. Imaging in extrapulmonary tuberculosis. Int J Infect Dis. 2017;56:237-47.

10. Geri G, Passeron A, Heym B, Arlet JB, Pouchot J, Capron L, Ranque B. Paradoxical reactions during treatment of tuberculosis with extrapulmonary manifestations in HIV-negative patients. Infection. 2013;41:537-43.

11. Grab J, Suarez I, van Gumpel E, Winter S, Schreiber F, Esser A, Holscher C, Fritsch M, Herb M, Schramm M, Wachsmuth L, Pallasch C, Pasparakis M, Kashkar H, Rybniker J. Corticosteroids inhibit Mycobacterium tuberculosis-induced necrotic host cell death by abrogating mitochondrial membrane permeability transition. Nat Commun. 2019;10:688.

12. Grebely J, Feld JJ, Applegate T, Matthews GV, Hellard M, Sherker A, Petoumenos K, Zang G, Shaw I, Yeung B, George J, Teutsch S, Kaldor JM, Cherepanov V, Bruneau J, Shoukry NH, Lloyd AR, Dore GJ. Plasma interferon-gamma-inducible protein-10
(IP-10) levels during acute hepatitis C virus infection. Hepatology. 2013;57:2124-34.

13. Hoel IM, Jorstad MD, Marijani M, Ruhwald M, Mustafa T, Dyrhol-Riise AM. IP-10 dried blood spots assay monitoring treatment efficacy in extrapulmonary tuberculosis in a low-resource setting. Sci Rep. 2019;9:3871.

14. Hong JY, Lee HJ, Kim SY, Chung KS, Kim EY, Jung JY, Park MS, Kim YS, Kim SK, Chang J, Cho SN, Kang YA. Efficacy of IP-10 as a biomarker for monitoring tuberculosis treatment. J Infect. 2014;68:252-8.

15. Horne DJ, Royce SE, Gooze L, Narita M, Hopewell PC, Nahid P, Steingart KR. Sputum monitoring during tuberculosis treatment for predicting outcome: systematic review and meta-analysis. Lancet Infect Dis. 2010;10:387-94.

16. Kamada A, Amishima M. 2017. QuantiFERON-TB((R)) Gold Plus as a potential tuberculosis treatment monitoring tool. Eur Respir J. 49.

17. Kerber R, Krumkamp R, Korva M, Rieger T, Wurr S, Duraffour S, Oestereich L, Gabriel M, Sissoko D, Anglaret X, Malvy D, May J, Zupanc TA, Munoz-Fontela C, Gunther S. Kinetics of soluble mediators of the host response in ebola virus disease. J Infect Dis. 2018;218:S496-503.

18. Khadge S, Banu S, Bobosha K, van der Ploeg-van Schip JJ, Goulart IM, Thapa P, Kunwar CB, van Meijgaarden KE, van den Eeden SJ, Wilson L, Kabir S, Dey H, Goulart LR, Lobato J, Carvalho W, Bekele Y, Franken KL, Aseffa A, Spencer JS, Oskam L, Otttenhoff TH, Hagge DA, Geluk A. Longitudinal immune profiles in type 1 leprosy reactions in Bangladesh, Brazil, Ethiopia and Nepal. BMC Infect Dis. 2015;15:477.

19. Ko TM, Kuo HC, Chang JS, Chen SP, Liu YM, Chen HW, Tsai FJ, Lee YC, Chen CH, Wu JY, Chen YT. CXCL10/IP-10 is a biomarker and mediator for Kawasaki disease. Circ Res. 2015;116:876-83.

20. Kruijshaar ME, Abubakar I. Increase in extrapulmonary tuberculosis in England and Wales 1999-2006. Thorax. 2009;64:1090-5.

21. Lanzafame M, Vento S. Tuberculosis-immune reconstitution inflammatory syndrome. J Clin Tuberc Other Mycobact Dis. 2016;3:6-9.

22. Lei J, Yin X, Shang H, Jiang Y. IP-10 is highly involved in HIV infection. Cytokine. 2019;115:97-103.

23. Mihret A, Bekele Y, Bobosha K, Kidd M, Aseffa A, Howe R, Walzl G. Plasma cytokines and chemokines differentiate between active disease and non-active tuberculosis infection. J Infect. 2013;66:357-65.

24. Nasiri MJ, Pormohammad A, Goudarzi H, Mardani M, Zamani S, Migliori GB, Sotgiu G. Latent tuberculosis infection in transplant candidates: a systematic review and meta-analysis on TST and IGRA. Infection. 2019;47:353-61.

25. Petruccioli E, Chiacchio T, Vanini V, Cuzzi G, Codecasa LR, Ferrarese M, Schinina V, Palmieri F, Ippolito G, Goletti D. Effect of therapy on Quantiferon-Plus response in patients with active and latent tuberculosis infection. Sci Rep. 2018;8:15626.

26. QIAGEN. 2016. QuantiFERON ${ }^{\circledR}$-TB Gold Plus (QFT®-Plus) ELISA Package Insert.

27. Qiu X, Tang Y, Zou R, Zeng Y, Yue Y, Li W, Qu Y, Mu D. Diagnostic accuracy of interferon-gamma-induced protein 10 for differentiating active tuberculosis from latent tuberculosis: A metaanalysis. Sci Rep. 2019;9:11408.

28. Qiu X, Xiong T, Su X, Qu Y, Ge L, Yue Y, Zeng Y, Li W, Hu P, $\mathrm{Mu}$ D. Accumulate evidence for IP-10 in diagnosing pulmonary tuberculosis. BMC Infect Dis. 2019;19:924.

29. Sharma SK, Soneja M. HIV \& immune reconstitution inflammatory syndrome (IRIS). Indian J Med Res. 2011;134:866-77.

30. Soni H, Bellam BL, Rao RK, Kumar PM, Mandavdhare HS, Singh H, Dutta U, Sharma V. Use of steroids for abdominal tuberculosis: a systematic review and meta-analysis. Infection. 2019;47:387-94. 
31. Sotgiu G, Saderi L, Petruccioli E, Aliberti S, Piana A, Petrone L, Goletti D. QuantiFERON TB Gold Plus for the diagnosis of tuberculosis: a systematic review and meta-analysis. J Infect. 2019;79:444-53.

32. Tadokera R, Meintjes G, Skolimowska KH, Wilkinson KA, Matthews K, Seldon R, Chegou NN, Maartens G, Rangaka MX, Rebe K, Walzl G, Wilkinson RJ. Hypercytokinaemia accompanies HIVtuberculosis immune reconstitution inflammatory syndrome. Eur Respir J. 2011;37:1248-59.

33. Tonby K, Ruhwald M, Kvale D, Dyrhol-Riise AM. IP-10 measured by Dry Plasma Spots as biomarker for therapy responses in Mycobacterium Tuberculosis infection. Sci Rep. 2015;5:9223.

34. van Hooij A, Boeters DM, Fat TMTK, van den Eeden SJF, Corstjens P, van der Helm-van Mil AHM, Geluk A. 2017. Longitudinal IP-10 serum levels are associated with the course of disease activity and remission in patients with rheumatoid arthritis. Clin Vaccine Immunol. 24.

35. Venkatappa TK, Punnoose R, Katz DJ, Higgins MP, Banaei N, Graviss EA, Belknap RW, Ho CS. 2019. Comparing
quantiFERON-TB gold plus with other tests to diagnose mycobacterium tuberculosis infection. J Clin Microbiol. 57.

36. Wergeland I, Pullar N, Assmus J, Ueland T, Tonby K, Feruglio S, Kvale D, Damas JK, Aukrust P, Mollnes TE, Dyrhol-Riise AM. IP-10 differentiates between active and latent tuberculosis irrespective of HIV status and declines during therapy. J Infect. 2015;70:381-91.

37. World Heal Organization (WHO). Global Tuberculosis Report 2019. 2019. https://www.who.int/teams/global-tuberculosis-progr amme/global-report-2019

38. Yang Y, Shen C, Li J, Yuan J, Wei J, Huang F, Wang F, Li G, Li Y, Xing L, Peng L, Yang M, Cao M, Zheng H, Wu W, Zou R, Li D, Xu Z, Wang H, Zhang M, Zhang Z, Gao GF, Jiang C, Liu L, Liu Y. Plasma IP-10 and MCP-3 levels are highly associated with disease severity and predict the progression of COVID-19. J Allergy Clin Immunol. 2020;146:119-127.e4.

\section{Affiliations}

\section{Isabelle Suárez ${ }^{1,2} \cdot$ Samuel Rohr ${ }^{1} \cdot$ Melanie Stecher ${ }^{1,2} \cdot$ Clara Lehmann $^{1,2,3} \cdot$ Sandra Winter $^{1,3} \cdot$ Norma Jung ${ }^{1,2}$. Vanessa Priesner ${ }^{1,2}$. Melanie Berger ${ }^{5}$ Christoph Wyen ${ }^{1,6} \cdot$ Max Augustin $^{1,3}$. Jakob J. Malin 1,2,3 . Julia Fischer ${ }^{1,2,3}$. Carola Horn $^{1,3} \cdot$ Florian Neuhann $^{4,9,10} \cdot$ Michael Püsken $^{8} \cdot$ Georg Plum $^{7} \cdot$ Gerd Fätkenheuer $^{1,2}$. Jan Rybniker ${ }^{1,2,3}$ (D)}

1 Division of Infectious Diseases, Department I of Internal Medicine, University Hospital of Cologne, University of Cologne, Kerpener Str. 62, 50937 Cologne, Germany

2 German Center for Infection Research (DZIF), Partner Site Bonn-Cologne, Cologne, Germany

3 Center for Molecular Medicine Cologne, University of Cologne, Cologne, Germany

4 Levy Mwanawasa Medical University (LMMU), Lusaka, Zambia

5 Department of Pneumology and Critical Care Medicine, Cologne-Merheim Hospital, Kliniken Der Stadt Köln GmbH, Witten/Herdecke University Hospital, Ostmerheimer Strasse 200, 51109 Cologne, Germany
6 Praxis Am Ebertplatz, Cologne, Germany

7 Institute for Medical Microbiology, Immunology and Hygiene, University Hospital of Cologne, Cologne, Germany

8 Institute of Diagnostic and Interventional Radiology, University Hospital of Cologne, Cologne, Germany

9 Institute of Global Health, University Hospital Heidelberg, Heidelberg, Germany

10 Municipal Health Authority Cologne, Cologne, Germany 